\title{
COMPARAÇÃO ENTRE MÉTODOS DE APROXIMAÇÃO NUMÉRICA UTILIZANDO O PROGRAMA MATLAB
}

Álvaro Pereira LOPES ${ }^{1}$

Campus Universitário de Abaetetuba - UFPA

alvarop14@ hotmail.com

Manuel de Jesus dos Santos COSTA ${ }^{2}$ Campus Universitário de Abaetetuba - UFPA

manuelsc@ufpa.br

Resumo: Este artigo de Iniciação Científica abordou, com o auxílio de códigos desenvolvidos em uma interface do programa Matlab, algumas comparações entre métodos de aproximação numérica e verificou qual deles se mostrou mais eficaz em determinadas situações. Foram apresentados os conceitos de interpolação polinomial, abordando a interpolação de Lagrange e de Newton, bem com a interpolação segmentada, abordando-se splines cúbicas. Algumas aplicações foram desenvolvidas. Primeiramente, compararam-se os métodos de Lagrange e Newton num exemplo prático: a determinação da largura de um rio para a construção de uma ponte. Em outra aplicação, foi analisado o comportamento do polinômio de Newton e da Spline Cúbica na interpolação da função de Runge. Ao analisar os resultados, foi possível concluir que, entre a interpolação de Lagrange e a de Newton, esta última é mais eficiente, uma vez que é mais leve em termos de esforço computacional, mas não é adequada para situações em que se têm muitos pontos de interpolação, ou seja, cujo polinômio interpolador tem o grau muito elevado. A spline cúbica se mostra eficiente nesses casos pelo fato de usar vários polinômios de grau baixo para formar a curva interpoladora.

Palavras-Chave: Aproximação Numérica. Interpolação. Splines. Auxílio Computacional.

\begin{abstract}
This Scientific Initiation article covered with the aid of codes developed an interface of the Matlab program some comparisons between numerical approximation methods and found which one was more effective in certain situations. The polynomial interpolation concepts were presented, addressing the interpolation of Lagrange and Newton, and segmented interpolation, approaching cubic splines. Some applications were developed. First, they compared the methods Lagrange and Newton a practical example, the determination of the width of a river for the construction of a bridge. In another application, it analyzed the Newton polynomial behavior and Cubic Spline interpolation in the Runge function. When analyzing the results, it was concluded that between the Lagrange interpolation and Newton latter is more efficient since it is lighter in terms of computational effort, but it is not suitable for situations where it has many interpolation points ie, where the polynomial interpolation is very high degree. The cubic spline is efficient in these cases shows that it uses several low degree polynomial to form the interpolating curve.
\end{abstract}

Keywords: Numerical approach. Interpolation. Splines. Computational assistance.

\footnotetext{
${ }^{1}$ Graduado em Matemática pela Universidade Federal do Pará - Campus Abaetetuba. Bolsista de Iniciação científica.

${ }^{2}$ Doutorado em Geofísica pela Universidade Federal do Pará, (2012). Professor Adjunto II da Universidade Federal do Pará.
} 


\section{Introdução}

O cálculo numérico é uma área de pesquisa matemática que consiste na utilização de métodos numéricos para dar soluções aproximadas a problemas de álgebra linear e não linear, estatística, cálculo diferencial e integral. Tais métodos foram desenvolvidos durante o século XIX, possibilitando o desenvolvimento desse novo ramo da matemática, antigamente chamado de matemática numérica.

A interpolação era largamente utilizada para o cálculo dos valores das funções transcendentes, como funções exponenciais, logarítmicas e trigonométricas. Em geral, possuíase somente uma tabela com os valores de tais funções para certo conjunto de argumentos e, quando era necessário o cálculo de algum valor não tabelado, fazia-se uso da interpolação.

Segundo Boyer (2010, pg. 439), na primeira metade do século XX, a história das máquinas de computação envolveu mais estatísticos, físicos e engenheiros elétricos que matemáticos. Enquanto lápis e papel continuavam a ser os instrumentos principais do matemático, máquinas de calcular de mesa e sistemas de cartões perfurados eram indispensáveis para negócios, bancos e para as ciências sociais. A régua de calcular se tornou o símbolo do engenheiro, e integradores de vários tipos eram usados por físicos, geodesistas e estatísticos. Essa situação mudou um tanto por volta de 1940 por causa do envolvimento de matemáticos no esforço de guerra.

Enquanto muitos matemáticos puros perseguiram o objetivo de substituir cálculos por ideias, engenheiros e matemáticos aplicados desenvolveram um instrumento que fez reviver o interesse por técnicas numéricas e algorítmicas e afetou fortemente a composição de muitos departamentos da matemática: o computador.(BOYER, 2010, pg. 439).

\section{Interpolação Polinomial}

Muitos problemas matemáticos não apresentam uma solução exata e, para tanto, faz-se uso de métodos que oferecem uma solução aproximada. De um modo geral, a interpolação é comumente utilizada quando $f(x)$ é conhecida apenas em alguns pontos no intervalo real $[a, b]$, e se deseja saber o seu valor num ponto diferente, mas ainda pertencente ao mesmo intervalo que os pontos conhecidos. Nesse caso, não se conhece a forma analítica da função e tais pontos conhecidos geralmente são obtidos em experimentos. Ou ainda, a forma analítica de $f(x)$ é muito complexa e apresenta um grau de dificuldade (ou mesmo impede) o uso de muitas operações como diferenciação e integração, de tal forma que se busca uma função $g(x)$ mais simples para substituí-la (RUGGIERO, 1996). 


\subsection{Conceito de Interpolação}

$\operatorname{Sejam} f(x)$ uma função definida em um intervalo $[a, b],(n+1)$ pontos distintos $x_{0}, x_{1}, \cdots, x_{n}$, chamados de nós da interpolação e $y_{i}=f\left(x_{i}\right)$ onde $i=0,1,2, \cdots, n$. Interpolar a função $f(x)$ consiste em aproximar esta função por um polinômio $P_{n}(x)$ de grau menor ou igual a $n$, de tal forma que este coincida com a função nesses pontos, isto é, $P_{n}\left(x_{i}\right)=$ $f\left(x_{i}\right)=y_{i}$, para $i=0,1, \cdots, n$.

Entretanto, ao se fazer a interpolação, espera-se que, para um $\bar{x} \neq x_{i} \operatorname{com} i=0,1, \cdots, n$, tenha-se $P_{n}(\bar{x}) \cong f(\bar{x})$ (ver Figura 1 ), ou seja, ao aproximar a função $f(x)$ pelo polinômio interpolador $P_{n}(x)$ comete-se um erro, dado por (ARENALES, 2010):

$$
E_{T}(\bar{x})=f(\bar{x})-P_{n}(\bar{x})
$$

Figura 1 - Exemplo de erro na interpolação

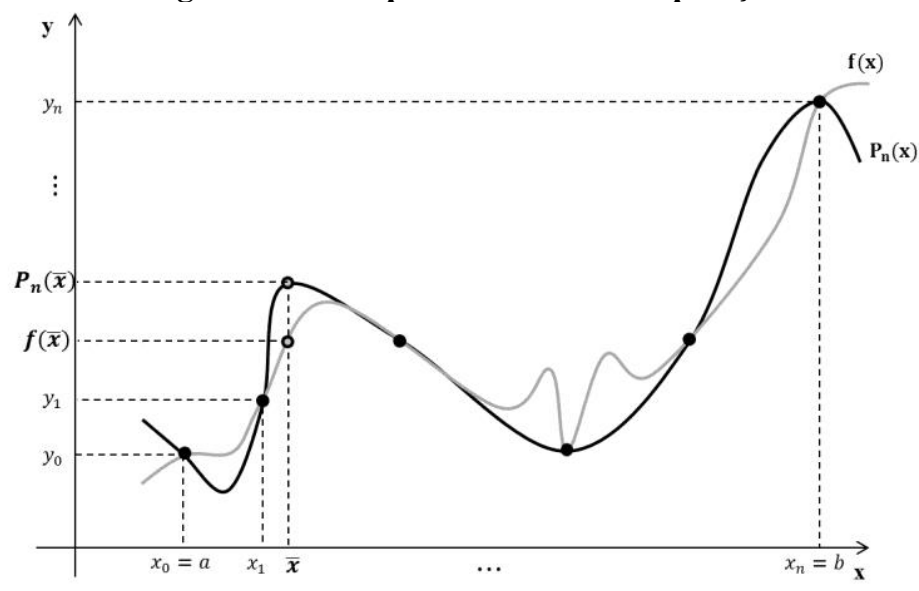

Fonte: Adaptado de ARENALES, 2010.

Por (1), fica evidente que somente é possível calcular uma estimativa para o erro da interpolação quando se conhece a expressão analítica da função $f(x)$, não sendo possível fazer o mesmo para o caso em que apenas são conhecidos alguns pontos da função.

\subsection{Métodos de Interpolação}

Sempre existirá (e será único) o polinômio interpolador $P_{n}(x)$ que satisfaça as condições da interpolação (RUGGIERO, 1996), no entanto há várias formas de se determiná-lo. 


\subsubsection{Interpolação de Lagrange}

Seja $f(x)$ definida em um intervalo $[a, b]$ contendo $(n+1)$ pontos distintos: $x_{0}, x_{1}, \cdots, x_{n}$, e $y_{i}=f\left(x_{i}\right), i=0,1, \cdots, n$. Representa-se o polinômio de grau $\leq n$ que interpola $f(x)$ em $x_{0}, x_{1}, \cdots, x_{n}$ pela forma:

$$
P_{n}(x)=y_{0} \cdot L_{0}(x)+y_{1} \cdot L_{1}(x)+\cdots+y_{n} \cdot L_{n}(x)
$$

onde os polinômios $L_{k}(x)$ são de grau ne definidos como:

$$
\begin{gathered}
L_{k}(x)=\frac{\left(x-x_{0}\right)\left(x-x_{1}\right) \cdots\left(x-x_{k-1}\right)\left(x-x_{k+1}\right) \ldots\left(x-x_{n}\right)}{\left(x_{k}-x_{0}\right)\left(x_{k}-x_{1}\right) \ldots\left(x_{k}-x_{k-1}\right)\left(x_{k}-x_{k+1}\right) \ldots\left(x_{k}-x_{n}\right)} \\
L_{k}\left(x_{i}\right)=\left\{\begin{array}{l}
0 \text { se } k \neq i \\
1 \text { se } k=i
\end{array}\right.
\end{gathered}
$$

Em suma, de maneira compacta, o polinômio de Lagrange é da forma (RUGGIERO, 1996):

$$
P_{n}(x)=\sum_{k=0}^{n} y_{k} L_{k}(x)=\sum_{k=0}^{n} y_{k} \prod_{\substack{j=0 \\ j \neq i}}^{n} \frac{\left(x-x_{\mathrm{j}}\right)}{\left(x_{k}-x_{\mathrm{j}}\right)}
$$

\subsubsection{Interpolação de Newton}

Seja $f(x)$ definida em um intervalo $[a, b]$ contendo $(n+1)$ pontos distintos: $x_{0}, x_{1}, \cdots, x_{n}$, e $y_{i}=f\left(x_{i}\right), i=0,1, \cdots, n$. Representa-se o polinômio de grau $\leq n$ que interpola $f(x)$ em $x_{0}, x_{1}, \cdots, x_{n}$ pela forma (ARENALES, 2010):

$$
\begin{aligned}
P_{n}(x)= & f\left[x_{0}\right]+f\left[x_{0}, x_{1}\right] \cdot\left(x-x_{0}\right)+f\left[x_{0}, x_{1}, x_{2}\right] \cdot\left(x-x_{0}\right) \cdot\left(x-x_{1}\right)+\cdots \\
& +f\left[x_{0}, x_{1}, \ldots, x_{n}\right] \cdot\left(x-x_{0}\right) \cdot\left(x-x_{1}\right) \ldots\left(x-x_{n-1}\right)
\end{aligned}
$$

onde os termos $f[$ ] são conhecidos como diferenças divididas, e são definidos, de acordo com sua ordem, como (ARENALES, 2010):

$$
\left\{\begin{array}{cc}
f\left[x_{i}\right]=f\left(x_{i}\right) & \text { ordem } 0 \\
f\left[x_{0}, x_{1}, \ldots, x_{n}\right]=\frac{f\left[x_{1}, x_{2}, \ldots, x_{n}\right]-f\left[x_{0}, x_{1}, \ldots, x_{n-1}\right]}{x_{n}-x_{0}} & \text { ordem } n
\end{array}\right.
$$

Notando que as diferenças divididas de ordem $k$ são calculadas a partir das diferenças divididas de ordem $k-1$, pode-se construir a tabela de diferenças divididas (ARENALES, 2010), como mostra o Quadro 1, a seguir. 
Quadro 1 - Tabela de diferenças divididas

\begin{tabular}{|c|c|c|c|c|c|c|}
\hline $\mathrm{x}$ & $\begin{array}{c}\text { Ordem } \\
0\end{array}$ & Ordem 1 & Ordem 2 & Ordem 3 & $\cdots$ & Ordem n \\
\hline$x_{0}$ & $f\left[x_{0}\right]$ & & & & & \\
\hline & & $f\left[x_{0}, x_{1}\right]$ & & & & \\
\hline$x_{1}$ & $f\left[x_{1}\right]$ & & $f\left[x_{0}, x_{1}, x_{2}\right]$ & & & \\
\hline & & $f\left[x_{1}, x_{2}\right]$ & & $f\left[x_{0}, x_{1}, x_{2}, x_{3}\right]$ & $\ddots$ & \\
\hline$x_{2}$ & $f\left[x_{2}\right]$ & & $f\left[x_{1}, x_{2}, x_{3}\right]$ & $\vdots$ & $f\left[x_{0}, x_{1}, x_{2}, \ldots, x_{n}\right]$ \\
\hline & & $f\left[x_{2}, x_{3}\right]$ & $\vdots$ & $f\left[x_{n-3}, x_{n-2}, x_{n-1}, x_{n}\right.$ & $\ddots$ & \\
\hline$x_{3}$ & $f\left[x_{3}\right]$ & $\vdots$ & $f\left[x_{n-2}, x_{n-1}, x_{n}\right]$ & & & \\
\hline$\vdots$ & $\vdots$ & $f\left[x_{n-1}, x_{n}\right]$ & & & & \\
\hline$x_{n}$ & $f\left[x_{n}\right]$ & & & & & \\
\hline
\end{tabular}

Fonte: Adaptado de RUGGIERO, 1996.

\section{Interpolação Segmentada}

Polinômios interpoladores de grau elevado podem produzir grandes oscilações nos extremos do intervalo, provocando o conhecido Fenômeno de Runge. Uma alternativa é interpolar $f(x)$ em grupos de poucos pontos, obtendo-se polinômios de grau menor, e impor condições para que a função de aproximação resultante seja contínua e tenha derivadas contínuas até certa ordem.

Figura 2 - Exemplo de interpolação segmentada de $\boldsymbol{f}(\boldsymbol{x})$

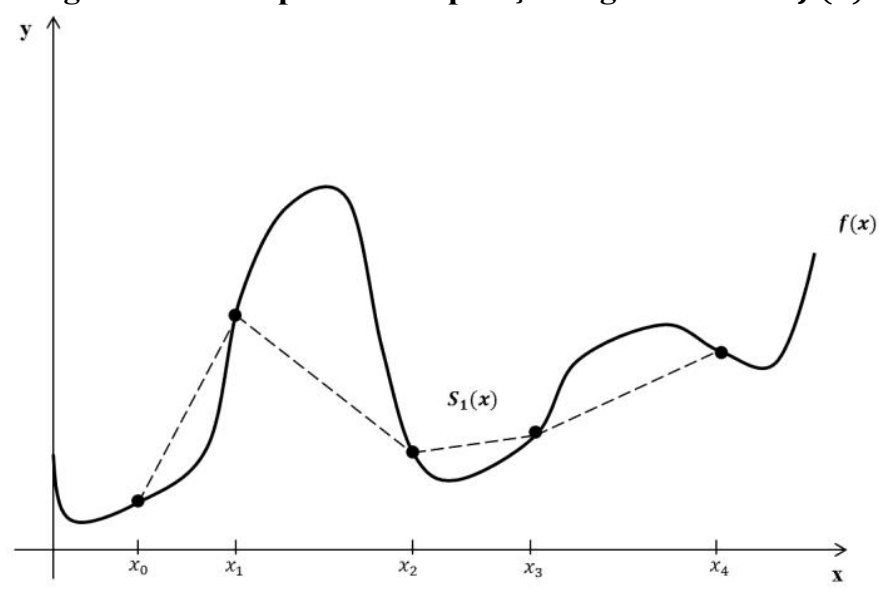

Fonte: RUGGIERO, 1996. 


\subsection{Função spline ${ }^{3}$}

Funções spline aproximam a função tabelada, em cada subintervalo $\left[\mathrm{x}_{\mathrm{i}}, \mathrm{x}_{\mathrm{i}+1}\right], i=$ $0,1, \cdots, n-1$, por um polinômio de grau $p$, com algumas imposições sobre a função spline. Considere a função $f(x)$ tabelada nos pontos $x_{0}<x_{1}<\cdots<x_{n}$. Uma função $S_{p}(x)$ é denominada spline interpolante de grau $p$ com nós nos pontos $x_{i}, i=0,1, \cdots, n$, se satisfaz as seguintes condições:

a) em cada subintervalo $\left[x_{i}, x_{i+1}\right], i=0,1, \cdots,(n-1), S_{p}(x)$ é um polinômio de grau $\mathrm{p}$, representado por $s_{p}(x)$;

b) $\quad S_{p}(x)$ é contínua e tem derivada contínua até ordem $(p-1)$ em $\left[x_{0}, x_{n}\right]$;

c) $\quad S_{p}\left(x_{i}\right)=f\left(x_{i}\right), i=0,1, \cdots, n$.

\subsubsection{Spline Cúbica Interpolante}

O fato de a spline cúbica ter a primeira e segunda derivada contínua garante que não tenha picos nem troque de curvatura abruptamente nos nós, e, por essa razão, esse tipo de spline é mais utilizado. Uma spline cúbica, $S_{3}(x)$ é uma função polinomial por partes, contínua, onde cada parte, $s_{k}(x)$, é um polinômio de grau 3 no intervalo $\left[x_{k-1}, x_{k}\right], k=1,2, \cdots, n$. Supondo que $f(x)$ esteja tabelada nos pontos $x_{i}, i=0,1,2, \cdots, n$ a função $S_{3}(x)$ é chamada spline cúbica interpolante de $f(x)$ nos nós $x_{i}, i=0,1,2, \cdots, n$ se existem $n$ polinômios de grau $3, s_{k}(x), k=$ $1,2, \cdots, n$, tais que as condições abaixo sejam satisfeitas:

i) $\quad S_{3}(x)=s_{k}(x) \operatorname{para} x \in\left[x_{k-1}, x_{k}\right], k=1,2, \cdots, n$;

ii) $\quad S_{3}\left(x_{i}\right)=f\left(x_{i}\right), i=1,2, \cdots, n$;

iii) $\quad s_{k}\left(x_{k}\right)=s_{k+1}\left(x_{k}\right), k=1,2, \cdots,(n-1)$;

iv) $\quad s^{\prime}{ }_{k}\left(x_{k}\right)=s^{\prime}{ }_{k+1}\left(x_{k}\right), k=1,2, \cdots,(n-1)$;

v) $\quad s^{\prime \prime}{ }_{k}\left(x_{k}\right)=s^{\prime \prime}{ }_{k+1}\left(x_{k}\right), k=1,2, \cdots,(n-1)$.

De forma genérica, os polinômios de grau $3 s_{k}(x)$ podem ser representados por (RUGGIERO, 1996):

$$
s_{k}(x)=a_{k}\left(x-x_{k}\right)^{3}+b_{k}\left(x-x_{k}\right)^{2}+c_{k}\left(x-x_{k}\right)+d_{k}, k=1,2, \cdots, n
$$

Assim, o cálculo de $S_{3}(x)$ exige a determinação de 4 coeficientes para cada $k$, num total de $4 n$ coeficientes, a saber: $a_{1}, b_{1}, c_{1}, d_{1}, a_{2}, b_{2}, c_{2}, d_{2} \cdots, a_{n}, b_{n}, c_{n}, d_{n}$. Para determiná-los, as

\footnotetext{
${ }^{3} \mathrm{~A}$ origem do nome spline vem de uma régua elástica usada em desenhos de engenharia, que pode ser curvada de forma a passar por um dado conjunto de pontos $\left(x_{i}, y_{i}\right)$. 
condições de $i$ ) $a v$ ) devem ser impostas, obtendo-se um total de $4 n-2$ condições. Portanto, há duas condições em aberto, citadas posteriormente.

Para facilitar a notação, usa-se:

$$
\begin{gathered}
h_{k}=x_{k}-x_{k-1} \\
s^{\prime \prime}{ }_{k}\left(x_{k}\right)=g_{k} \\
f\left(x_{k}\right)=y_{k}
\end{gathered}
$$

obtendo-se as fórmulas para determinar os coeficientes de $s_{k}(x)$

$$
\begin{gathered}
a_{k}=\frac{g_{k}-g_{k-1}}{6 h_{k}} \\
b_{k}=\frac{g_{k}}{2} \\
c_{k}=\left[\frac{y_{k}-y_{k-1}}{h_{k}}+\frac{2 h_{k} g_{k}+g_{k-1} h_{k}}{6}\right] \\
d_{k}=y_{k}
\end{gathered}
$$

em função de $g_{j}=s^{\prime \prime}{ }_{j}\left(x_{j}\right), j=0,1, \cdots, n$, para $k=1,2, \cdots, n$.

Ao final, por meio do sistema de equações lineares com $(n-1)$ equações $(k=$ $1, \cdots,(n-1))$ e $(n+1)$ incógnitas: $g_{0}, g_{1}, \cdots, g_{n-1}, g_{n}$ e, portanto, indeterminado, na forma $A \cdot X=B$, abaixo:

$$
h_{k} g_{k-1}+2\left(h_{k}+h_{k+1}\right) g_{k}+h_{k+1} g_{k+1}=6\left(\frac{y_{k+1}-y_{k}}{h_{k+1}}-\frac{y_{k}-y_{k-1}}{h_{k}}\right)
$$

onde

$$
\begin{aligned}
& X=\left(g_{0}, g_{1}, \cdots, g_{n-1}, g_{n}\right)^{T}
\end{aligned}
$$

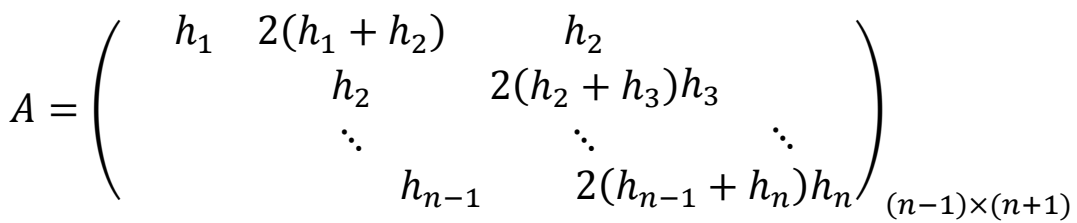

$\mathrm{e}$

$$
B=6\left(\begin{array}{ccc}
\frac{y_{2}-y_{1}}{h_{2}} & -\frac{y_{1}-y_{0}}{h_{1}} \\
\frac{y_{3}-y_{2}}{h_{3}} & - & \frac{y_{2}-y_{1}}{h_{2}} \\
\vdots & \vdots \\
\frac{y_{n}-y_{n-1}}{h_{n}} & -\frac{y_{n-1}-y_{n-2}}{h_{n-1}}
\end{array}\right)_{(n-1) \times 1}
$$


É necessário impor mais duas condições para poder resolvê-lo de forma única. De posse da solução, é possível determinar $a_{k}, b_{k}, c_{k}, e d_{k}$, para cada $s_{k}(x)$.

\section{Aplicações}

A fim de avaliar qual dos métodos apresentados neste trabalho é o mais eficaz, quando se analisam determinados aspectos de seu funcionamento e sua eficiência na resolução de um problema, apresenta-se a seguir um exemplo prático e duas comparações, executadas com o auxílio de códigos desenvolvidos no programa Matlab.

\subsection{Determinação da largura de um rio}

A PA 151 é uma rodovia importante que liga as cidades de Belém, Barcarena, Abaetetuba, Igarapé-Miri, Mocajuba, Baião e Cametá, além de vilas. Ao longo do percurso da rodovia, há duas travessias de balsas: uma sobre o Rio Meruú (divisa natural entre os municípios de Mocajuba e Igarapé-Miri) e outra sobre o Rio Igarapé-Miri (que banha a frente da cidade de mesmo nome), cujo preço do serviço de transporte para veículos é de $\mathrm{R} \$ 6,91^{4}$.

De um ponto de vista geral, seria de utilidade imprescindível a construção de uma ponte para se realizar estas travessias. Com o objetivo de ratificar os dados de medições de equipamentos, deseja-se saber a largura do rio Igarapé-Miri para esta construção, por meio de interpolação.

Utilizaram-se, como base dados hipotéticos, imagens de satélite. A partir de uma linha reta, X, próxima a uma das margens, foram medidas distâncias entre esta linha reta e as duas margens do rio, de 100 em 100 metros, a partir de um ponto tomado como origem de X. O resultado dessas medições está registrado na Tabela 1. O objetivo da interpolação é determinar o valor aproximado da largura do rio Igarapé-Miri no ponto que dista 350 metros da origem (tomado em X). A seguir, a Figura 3 e a Figura 4 mostram a situação descrita anteriormente.

Figura 3 - Imagem por satélite do Rio Igarapé-Miri

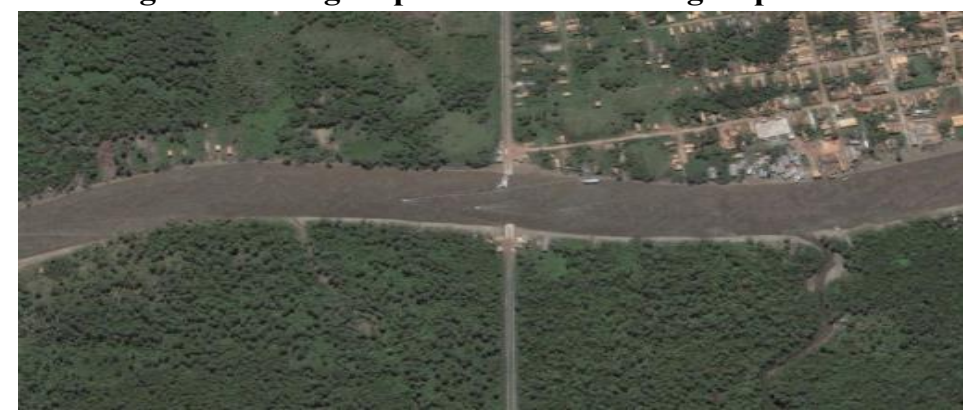

Fonte: https://www.google.com.br/maps/@-1.9808206,-48.9731465,20z

${ }^{4}$ Valor de jan./2011. 
Visto que o rio Igarapé-Miri banha a frente do município de mesmo nome, adotou-se para uma melhor compreensão a notação de M1 para a margem oposta ao município (margem inferior das figuras), e M2 para a margem que faz parte de Igarapé-Miri (margem superior das figuras). Com relação à Figura 3, a linha reta X se encontra abaixo de M1, como visto abaixo:

Figura 4 - Representação gráfica do sistema de eixos cartesianos orientado pela linhareta $X$

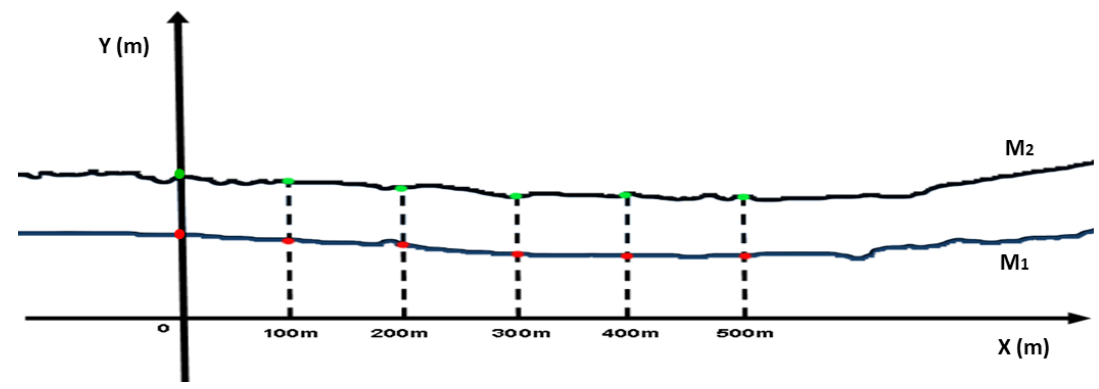

Fonte: Autoria própria

Tabela 1 - Resultado das medições entre a linha reta X e as margens M1 e M2 (em m)

\begin{tabular}{c|c|c|c|c|c|c}
\hline Indicador & \multicolumn{6}{|c}{ Medições } \\
\hline X & 0 & 100 & 200 & 300 & 400 & 500 \\
\hline M1 & 137,65 & 128,35 & 121,54 & 106,88 & 104,26 & 101,59 \\
M2 & 236,91 & 230,48 & 226,51 & 214,25 & 204,96 & 202,53 \\
\hline
\end{tabular}

Fonte: Autoria própria

Como já mencionado, a interpolação de Lagrange ou a interpolação de Newton determinam o mesmo polinômio interpolador. Dessa forma, optou-se pelo último método citado para solucionar o problema, utilizando uma rotina computacional desenvolvida em uma interface do programa Matlab, cujos resultados são apresentando na Figuras 5.

Figura 5 - Representação gráfica das distâncias entre a linha reta X e as margens M1 e M2 do rio, incluindo os pontos onde a ponte passaria (valores em metros)

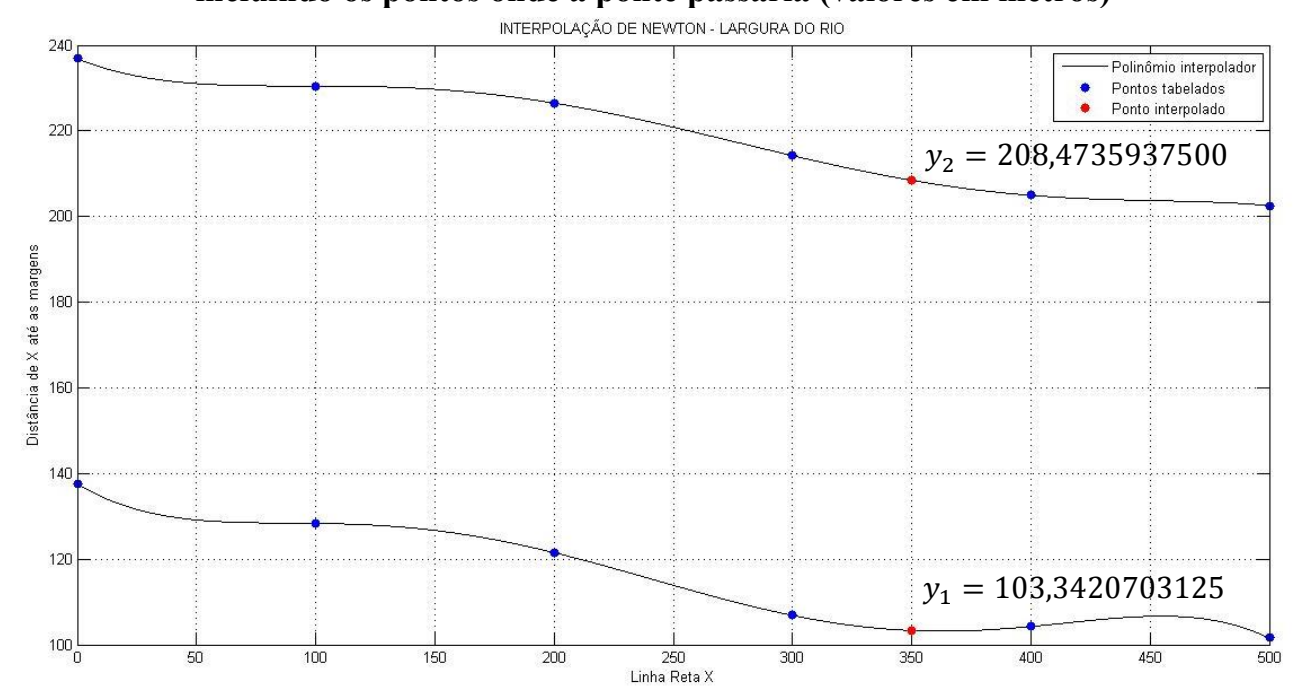

Fonte: Autoria própria 
Com base nos valores obtidos pela interpolação de Newton representada na Figura 5, a largura do rio Igarapé-Miri no local da construção da ponte é aproximadamente igual a:

$$
y_{2}-y_{1}=208,4735937500-103,3420703125=105,131473046875 \mathrm{~m}
$$

A ponte em questão já se encontra em construção. Entretanto, ressalta-se que a ratificação com base em dados reais da largura do rio Igarapé-Miri não pôde ser feita por dificuldades no acesso aos dados oficiais da construção da ponte. Dessa forma, mostra-se a validade desta aplicação para determinar o valor procurado, cabendo mudanças nos valores dos pontos da Tabela 1 para a situação real.

\subsection{Comparação entre os métodos de Lagrange e Newton}

Utilizou-se os dados da Tabela 1 relativos à margem M1 do rio para analisar qual dos dois métodos de interpolação polinomial (Lagrange ou Newton) é o mais eficiente na determinação do polinômio interpolador. Comparando o tipo e a quantidade das operações fundamentais da aritmética necessárias para solucionar o problema proposto na aplicação anterior, geraram-se os gráficos da Figura 6 e a Tabela 2:

Figura 6 - Comparação percentual entre os tipos de operações fundamentais da aritmética nos métodos de interpolação de Lagrange e de Newton

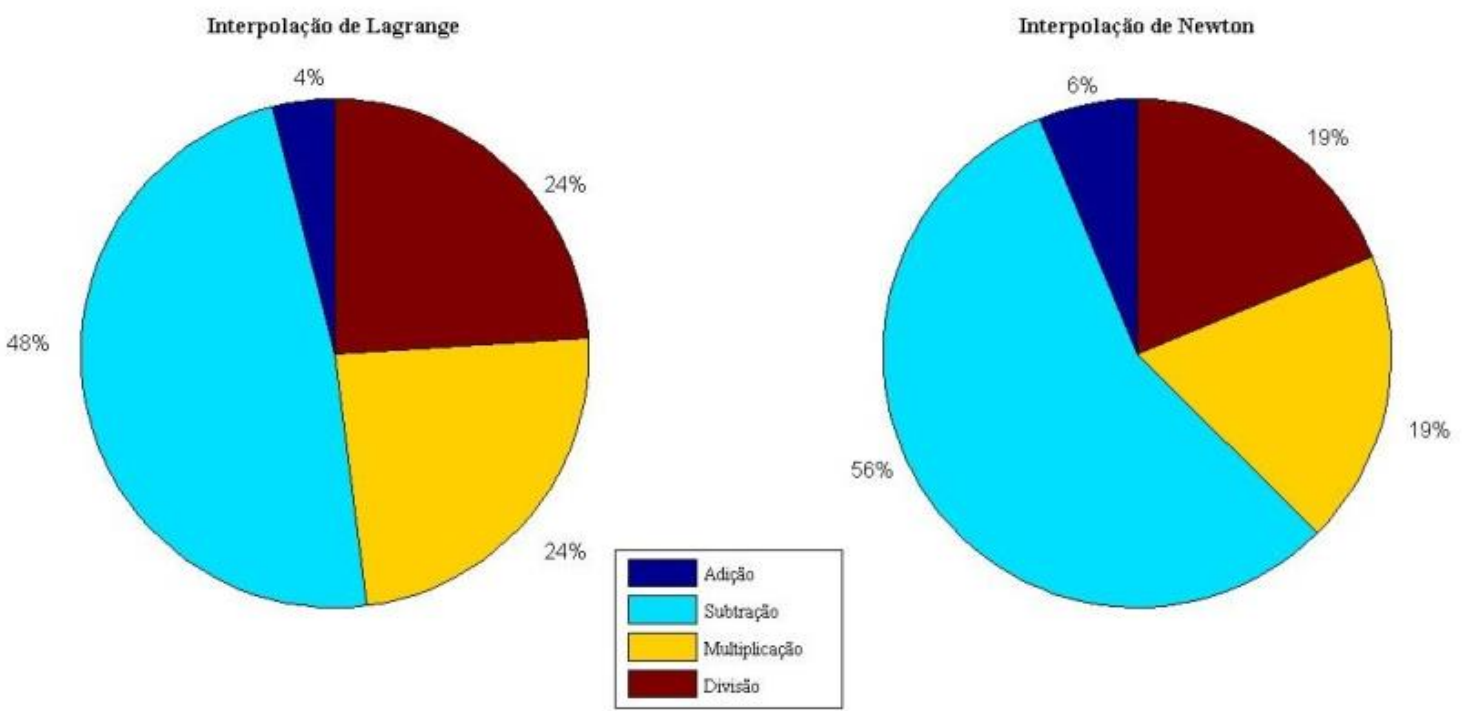

Fonte: Autoria própria

A análise da Figura 6 permite destacar alguns pontos em comum e alguns pontos diversos entre a interpolação de Lagrange e a Interpolação de Newton.

Dentre os pontos em comum, pode-se citar:

- A adição representa a menor porcentagem dentre as operações fundamentais; 
- Multiplicação e divisão aparecem com a mesma porcentagem;

- A subtração representa a maior porcentagem dentre as operações fundamentais;

Entretanto, divergências evidentes são:

- A adição e subtração representam uma porcentagem menor na interpolação de Lagrange em relação à de Newton;

- A multiplicação e divisão representam uma porcentagem menor na interpolação de Newton em relação à de Lagrange.

Já a análise numérica, apresentada na Tabela 2, permite estabelecer com maior clareza qual o método de interpolação que apresenta maiores vantagens no que diz respeito ao esforço computacional, e consequentemente, ao tempo necessário para realizar um cálculo.

Tabela 2 - Contagem numérica dos tipos de operações aritméticas na interpolação de Lagrange e na interpolação de Newton

\begin{tabular}{c|c|c}
\hline & Interpolação de Lagrange & Interpolação de Newton \\
\hline Adição & 5 & 5 \\
Subtração & 60 & 45 \\
Multiplicação & 30 & 15 \\
Divisão & 30 & 15 \\
\hline Total & 125 & 80 \\
\hline
\end{tabular}

Fonte: Autoria própria

Com exceção da adição, percebe-se que todas as outras operações são mais requisitadas na interpolação de Lagrange. Isso influencia diretamente o número total de operações feitas para determinar a mesma interpolação, tornando o método de Lagrange mais "pesado" em termos de esforço computacional. Dessa forma, entre as duas formas de se determinar o polinômio interpolador abordadas neste trabalho à interpolação de Newton requer um esforço computacional menor e, portanto, é mais eficaz.

\subsection{Comportamento do polinômio de Newton e da spline cúbica na aproximação da função de Runge}

A título de conhecimento, ao final deste artigo, encontra-se o algoritmo desenvolvido com base na linguagem de programação do Matlab para determinar a spline cúbica. Ressaltase que, com esses comandos e com as devidas alterações para cada tipo de linguagem computacional, se prestam para determinar qualquer spline cúbica pretendida.

Esta comparação entre a interpolação polinomial de Newton e a interpolação segmentada por spline Cúbica analisa os seus comportamentos na aproximação da função de Runge. O objetivo é verificar o que ocorre com as funções à medida que se aumenta o número de pontos de interpolação de forma considerável, ratificando-se assim o Fenômeno de Runge. 
Fez-se várias interpolações, variando de 3 pontos até 25 pontos interpolados, e analisou-se a maior variação do erro, considerando-se somente os pontos pertencentes ao intervalo [-1, -0.8], por meio da fórmula (1). A relação entre o número de pontos interpolados e o valor do erro é vista na Tabela 3.

Tabela 3 - Relação entre o número de pontos de interpolação e a maior variação do erro

\begin{tabular}{|c|c|c|}
\hline & $\begin{array}{l}\text { Interpolação de } \\
\text { Lagrange }\end{array}$ & Spline Cúbica \\
\hline $\begin{array}{l}\text { Número de } \\
\text { pontos }\end{array}$ & $\begin{array}{l}\text { Maior variação do } \\
\text { erro próximo aos } \\
\text { extremos }\end{array}$ & $\begin{array}{l}\text { Maior variação } \\
\text { do erro próximo } \\
\text { aos extremos }\end{array}$ \\
\hline 3 & -0.3257918552036 & -26.43 \\
\hline 4 & -0.0712669683258 & -5.99 \\
\hline 5 & 0.4381338742394 & 9.52 \\
\hline 6 & 0.1094839309876 & 3.09 \\
\hline 7 & -0.6169479236760 & -1.91 \\
\hline 8 & -0.1715775505739 & -0.94 \\
\hline 9 & 1.0451739117837 & 0.20 \\
\hline 10 & 0.3002939878926 & 0.15 \\
\hline 11 & -1.9156430502193 & -0.06 \\
\hline 12 & -0.5567554873096 & -0.06 \\
\hline 13 & 3.6632621433137 & -0.03 \\
\hline 14 & 1.0700405885115 & -0.02 \\
\hline 15 & -7.1948811072331 & -0.02 \\
\hline 16 & -2.1075518461337 & -0.02 \\
\hline 17 & 14.3938512850034 & -0.02 \\
\hline 18 & 4.2238338577280 & -0.01 \\
\hline 19 & -29.1856486754932 & -0.01 \\
\hline 20 & -8.5785651072100 & -0.01 \\
\hline 21 & 59.8223087107278 & -0.01 \\
\hline 22 & 17.6020221123122 & -0.01 \\
\hline 23 & $\begin{array}{c}- \\
123.6166738204971\end{array}$ & -0.01 \\
\hline 24 & -36.4007343161108 & -0.01 \\
\hline 25 & 257.2129123345954 & -0.01 \\
\hline
\end{tabular}

Fonte: Autoria própria

Nas Figuras (7 e 8), os valores da Tabela (3) podem ser visualizados, mostrando a oscilação das maiores variações do erro no polinômio de Newton:

Essas imagens evidenciam que a spline cúbica converge muito mais rapidamente e eficientemente para a função de Runge do que o polinômio de Newton. Assim como Runge constatou, o fato de a spline ser uma interpolação por partes, em que cada segmento entre os pontos interpolados é um polinômio de grau 3, é mais eficiente na diminuição do erro de interpolação do que aumentar o grau do polinômio. 
Figura 7 - Oscilação das maiores variações do erro na interpolação polinomial

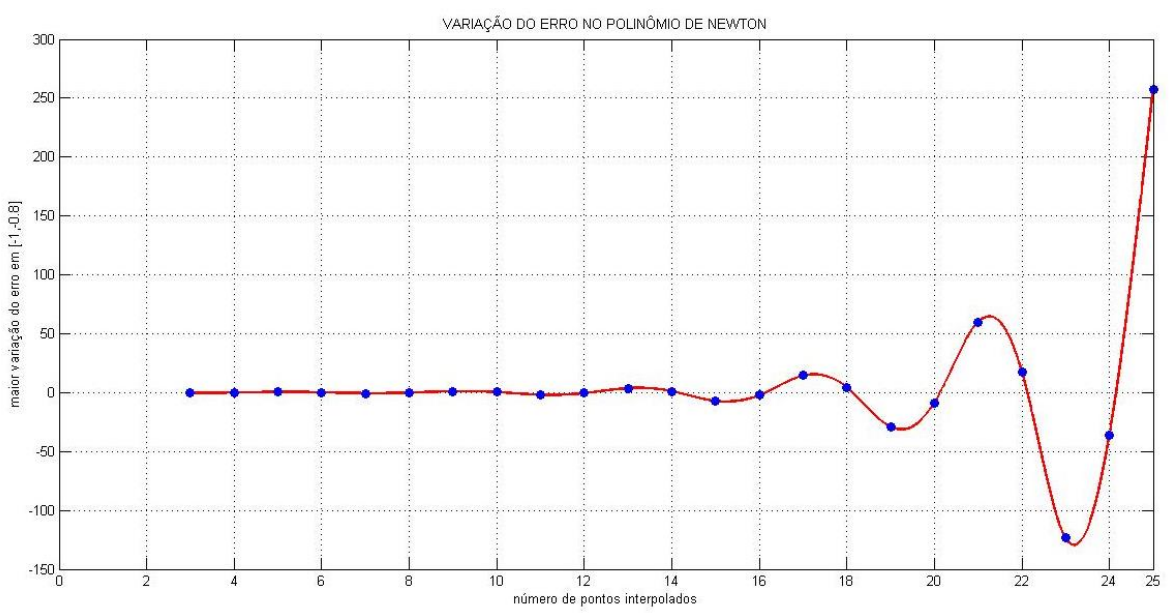

Fonte: Autoria própria

Figura 8 - Oscilação das maiores variações do erro na interpolação segmentada

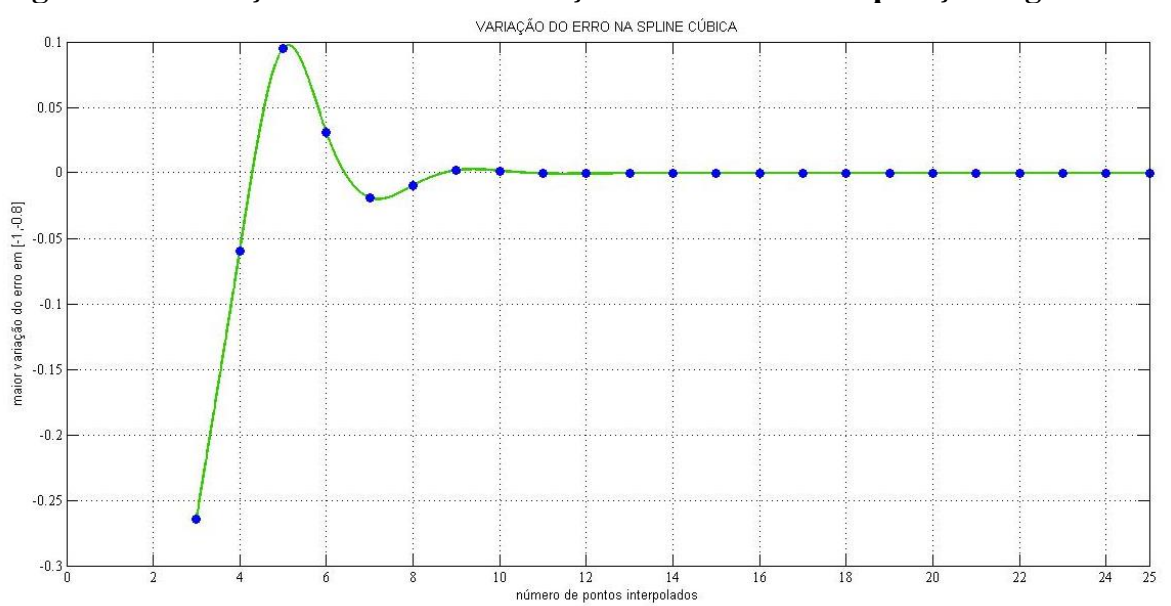

Fonte: Autoria própria

\section{Considerações finais}

Neste trabalho, foram comparados alguns métodos de aproximação numérica utilizando o programa Matlab, com o objetivo de analisar qual o mais eficiente em determinadas situações. Os métodos de interpolação polinomial (Lagrange e Newton) e interpolação segmentada (Spline) revisados aqui são amplamente comentados na literatura científica. Ao avaliar os resultados das aplicações, foi possível concluir que, entre a interpolação de Lagrange e a de Newton, esta última é mais eficiente, uma vez que é mais leve em termos de esforço computacional, mas não é adequada para situações em que se tem muitos pontos de interpolação, ou seja, cujo polinômio interpolador tem o grau muito elevado. A spline cúbica se mostra eficiente nesses casos pelo fato de usar vários polinômios de grau baixo para formar a curva interpoladora. Além disso, verificou-se a importância do auxílio computacional como ferramenta a tais aplicações, em casos que seria impraticável fazer tantos cálculos manualmente, e obter uma confirmação visual dos estudos por meio dos gráficos. 


\section{Referências}

ARENALES, Selma; DAREZZO, Artur. Aproximação de funções. In: Cálculo

Numérico: aprendizagem com apoio de software. São Paulo: Cengage Learning, 2010. Cap. IV, pg 127-188.

BECKER, Alex J. et al. Noções Básicas de Programação em Matlab. Santa Maria: UFSM, 2010.

BOYER, Carl B. História da matemática. Revista por Uta C. Merzbach. Prefácio de Isaac Asimov. Tradução de Elza F. Gomide. 3. ed. São Paulo: Blucher, 2010.

RUGGIERO, Márcia A. Gomes; LOPES, Vera Lúcia da Rocha. Interpolação. In:

Cálculo numérico: aspectos teóricos e computacionais. 2. ed. São Paulo: Person Makron Books, 1996. Cap. V,pg 211-267. 